\title{
Effects of adatoms and physisorbed molecules on the physical properties of antimonene
}

\author{
O. Üzengi Aktürk, ${ }^{1,2,3}$ E. Aktürk, ${ }^{2,3,4}$ and S. Ciraci ${ }^{3, *}$ \\ ${ }^{1}$ Department of Electric and Electronic Engineering, Adnan Menderes University, 09100 Aydin, Turkey \\ ${ }^{2}$ Nanotechnology Application and Research Center, Adnan Menderes University, Aydin 09010, Turkey \\ ${ }^{3}$ Department of Physics, Bilkent University, 06800 Ankara, Turkey \\ ${ }^{4}$ Department of Physics, Adnan Menderes University, 09100 Aydın, Turkey
}

(Received 30 November 2015; revised manuscript received 31 December 2015; published 27 January 2016)

\begin{abstract}
A recent study predicted that a $2 \mathrm{D}$ single layer of antimony in buckled honeycomb as well as asymmetric washboard structures, named antimonene, is stable at high temperature and displays semiconducting properties. Based on first-principles, spin-polarized density functional calculations, we investigated chemisorption of selected adatoms and physisorption of molecules on two antimonene phases. Since adspecies-adspecies interaction is minimized by using large supercells, our results mimic the effects of isolated, single adatoms or molecules. We found that molecules such as $\mathrm{H}_{2}, \mathrm{O}_{2}$, and $\mathrm{H}_{2} \mathrm{O}$ neither form strong chemical bonds nor dissociate; they are physisorbed with a weak binding energy without affecting the properties of antimonene. The adatoms, such as $\mathrm{H}, \mathrm{Li}, \mathrm{B}, \mathrm{C}, \mathrm{N}, \mathrm{O}, \mathrm{Al}, \mathrm{In}, \mathrm{Si}, \mathrm{P}, \mathrm{Cl}, \mathrm{Ti}$, As, and Sb, are chemisorbed with significant binding energy, whereby the atomic and electronic structures are modified locally. Boron and carbon adatoms are implemented into buckled antimonene crystal leading to a local reconstruction of the crystal. Nitrogen gives rise to Stone-Wales type defects. The localized states originating from adatoms give rise to diversity of electronic structure. The lowest conduction and highest valence bands of antimonene in asymmetric washboard structures have very high curvature. Once combined with adatom states, these bands offer a variety of features. Specific adatoms lead to spin polarization, attain magnetic moments, and can attribute a half-metallic character to antimonene.
\end{abstract}

DOI: 10.1103/PhysRevB.93.035450

\section{INTRODUCTION}

Our recent theoretical study [1] has shown that antimony (Sb) can form two different, single-layer (SL) 2D crystalline structures like silicene [2-5] and black phosphorene [6]. These are a buckled honeycomb structure (buckled, Bu antimonene or $\mathrm{Bu}-\mathrm{Sb}$ ) and an asymmetric washboard structure (aW antimonene or $\mathrm{aW}-\mathrm{Sb}$ ). These two suspended phases have been found to be semiconductors and to maintain their stability at temperatures as high as $1000 \mathrm{~K}$. Additionally their armchair and zigzag nanoribbons have displayed electronic properties, which may be interesting in 2D flexible electronics. These theoretical findings concerning antimonene phases are corroborated by the fabrication of very thin antimony films [7-9].

Previous experimental and theoretical studies have proven that bare SL structures can be functionalized through adsorption of adatoms to attain crucial physical and chemical properties for diverse applications. For example, while graphene is inert to $\mathrm{H}_{2}$ molecules, each $\mathrm{Ti}$ or $\mathrm{Li}$ adatom adsorbed to graphene can hold $4-5 \mathrm{H}_{2}$ molecules providing an excellent high-capacity medium for hydrogen storage [10-12]. Similarly, the graphene nanoribbon attains half metallicity through the adsorption of Fe adatoms [13]. Moreover, those properties have been shown to be strongly dependent on the coverage of adatoms to lead to tunable electronic properties. Accordingly, these unusual electronic, magnetic, and chemical properties achieved through the adsorption of foreign atoms to SL honeycomb structures have provided important applications and hence have augmented the interest in bare structures. In this respect, while bare antimonene phases are predicted to be semiconducting

*ciraci@fen.bilkent.edu.tr nanostructures, their physical properties are expected to be modified to a large extend by the adsorption of adatoms.

In this paper we provide an analysis of the effects of chemisorbed adatoms and physisorbed molecules on the physical properties of antimonene. Here we consider the effect of single $\mathrm{H}, \mathrm{Li}, \mathrm{B}, \mathrm{C}, \mathrm{N}, \mathrm{O}, \mathrm{Al}, \mathrm{In}, \mathrm{Si}, \mathrm{P}, \mathrm{Cl}, \mathrm{Ti}, \mathrm{As}$, and $\mathrm{Sb}$ adatoms, which were found to modify the properties of bare graphene [13-15], silicene [16,17], phosphorene [18], and $\mathrm{MoS}_{2}$ [19] and give rise to diverse properties. In the past, physisorption of $\mathrm{H}_{2}, \mathrm{O}_{2}$, and $\mathrm{H}_{2} \mathrm{O}$ molecules has been of interest because of the oxidation-deoxidation process [20-22], possible hydrogen evaluation reaction [23], and hydrogen storage [10-12]. Owing to a large number atoms and molecules included in our study, we treated only the effects of adsorption of single (isolated) adatoms and physisorbed single molecules on the atomic structure and electronic properties of antimonene using a realistic method. Coverage-dependent properties are beyond the scope of the present paper.

Important predictions of our paper which are obtained using first-principles density functional theory [24] (DFT) are summarized: (i) $\mathrm{H}_{2}, \mathrm{O}_{2}$, and $\mathrm{H}_{2} \mathrm{O}$ molecules neither form strong chemical bonds nor dissociate; they are physisorbed with a weak binding energy without affecting the properties of antimonene significantly. (ii) On the other hand, single adatoms, H, Li, B, C, N, O, Al, In, Si, P, Cl, Ti, As, and Sb, are adsorbed with significant binding energy. Some of these adatoms are implemented into the antimonene crystal through local reconstructions; nitrogen can form Stone-Wales type defects. (iii) Most of the adatoms treated here have localized states near the band edges and attribute crucial electronic properties to antimonene. Some of the adatoms give rise to spin polarization and attain magnetic moments.

The organization of the paper is as follows: In Sec. II, we summarize the atomistic model and the method of our study by 
describing crucial parameters of calculations. In Sec. III, we present a brief summary on the properties of SL antimonene phases predicted in our previous study [1]. This way one can contrast the modified properties of adatoms adsorbed to antimonene phases with those of bare ones. Section IV is devoted to the analysis of the adsorption of adatoms, including the equilibrium atomic structure, corresponding binding energy, electronic structure, and magnetic state of single adatoms adsorbed to $\mathrm{Bu}-\mathrm{Sb}$ and $\mathrm{aW}-\mathrm{Sb}$. Section $\mathrm{V}$ provides information for the weak interaction of antimonene with selected molecules such as $\mathrm{H}_{2}, \mathrm{O}_{2}$, and $\mathrm{H}_{2} \mathrm{O}$. In Sec. VI our conclusions are summarized.

\section{ATOMISTIC MODEL AND METHOD OF CALCULATIONS}

Adsorption of the single adatoms to $\mathrm{Bu}-\mathrm{Sb}$ and $\mathrm{aW}-\mathrm{Sb}$ is treated within supercell geometry using periodic boundary conditions. Each supercell is constructed from a $(n \times n)$ primitive cell of $\mathrm{Bu}-\mathrm{Sb}$ and aW-Sb. One adatom is placed at the same position of each supercell, so that adsorbed adatoms form a regular array. Here the $2 \mathrm{D}$ translation vectors of the supercell are $\mathbf{R}_{s_{1}}=n\left(\mathbf{R}_{1}\right)$ and $\mathbf{R}_{s_{2}}=n\left(\mathbf{R}_{2}\right)$ in terms of the translation vectors of the primitive unit cells $\mathbf{R}_{1}$ and $\mathbf{R}_{2}$ of $\mathrm{Bu}-\mathrm{Sb}$ and $\mathrm{aW}-\mathrm{Sb}$. The coupling between adjacent adatoms decreases with increasing $n$. Here we considered $n=5$ supercells for $\mathrm{Bu}-\mathrm{Sb}$ and $n=4$ supercells for aW-Sb phases. Physisorbed molecules also are treated using similar supercells. In these supercells the smallest separations between adatoms or physisorbed molecules on the $\mathrm{Bu}-\mathrm{Sb}$ and $\mathrm{aW}$ Sb phases attained distances $l_{\mathrm{Bu}}=20 \AA$ and $l_{\mathrm{aW}}=19 \AA$, respectively.

Our models of supercells for $\mathrm{Bu}-\mathrm{Sb}$ and $\mathrm{aW}-\mathrm{Sb}$ phases and possible adsorption sites are described in Fig. 1. The equilibrium structure of each adspecies and corresponding atomic configuration including the substrate is determined fol-
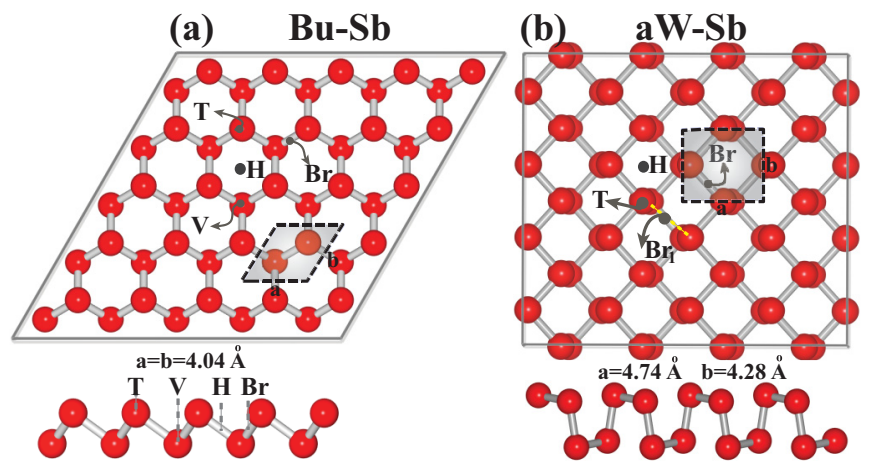

FIG. 1. (a) Top and side views of the atomic configuration of the $(5 \times 5)$ supercells of $\mathrm{Bu}-\mathrm{Sb}$ phase used to treat the adsorbed single adatom. 2D hexagonal primitive unit cell is delineated by dashed lines. Possible adsorption sites are the top site $(\mathrm{T})$, where the adatom is on top of the host $\mathrm{Sb}$ atoms; the bridge site $(\mathrm{Br})$, where the adatom is above the center of the $\mathrm{Sb}$-Sb bond; the hollow site $(\mathrm{H})$, where the adatom is located above the center of hexagons of the host $\mathrm{Sb}$ atoms; the valley site (V), where the adatom is placed on top the low-buckled host $\mathrm{Sb}$ atoms. (b) Top and side views of the atomic configuration of the $(4 \times 4)$ supercells of aW-Sb. 2D rectangular primitive unit cell is also shown. $\left|\mathbf{R}_{1}\right|=a$ and $\left|\mathbf{R}_{2}\right|=b$. lowing a comprehensive optimization process. First adatoms or molecules are placed to all possible sites described in Fig. 1 at a distant height from the SL substrate. Subsequently, all atomic positions including the height of adatom or molecules and atomic positions of substrate atoms are relaxed to attain the minimum total energy, $E_{T}[$ substrate $+A]$. Here $A$ denotes adatom or molecule. Then the binding energy is obtained from the expression $E_{b}=E_{T}$ [substrate] $+E_{T}[A]-$ $E_{T}$ [substrate $\left.+A\right]$ in terms of the total spin-polarized energies of the free adatom (molecule), of bare substrate, and of the system of adatom adsorbed (molecule physisorbed) to the substrate. Here the positive value of $E_{b}$ indicates that the binding of adatom or molecule to antimonene is favorable for a given geometry. Since the initial configuration relative to the substrate is crucial for physisorbed molecules, various orientations have been tested to achieve the highest binding energy. It should be noted that adatoms, which have minute couplings with nearest adatoms in adjacent supercells for large $n$, can mimic the chemisorption of a single atom on a large antimonene substrate. On the other hand, adatoms treated using small $n$ lead to high coverage resulting in a specific decoration, whereby the interaction between a single adatom and substrate and hence the physical properties thereof are modified significantly depending on the coverage. For example, at high coverage the localized electronic states originating from adatoms form energy bands.

Our theoretical analysis and predictions are obtained from first-principles pseudopotential calculations based on the spin-polarized DFT within the generalized gradient approximation including van der Waals corrections [25]. Here we used projector-augmented wave (PAW) potentials [26] and the exchange-correlation potential is approximated with the Perdew-Burke-Ernzerhof (PBE) functional [27]. Numerical calculations were carried out using the VASP software [28]. A plane-wave basis set with kinetic energy cutoff is taken to be $\hbar^{2}|\mathbf{k}+\mathbf{G}|^{2} / 2 m=550 \mathrm{eV}$. The Brillouin zone (BZ) was sampled in the k space within the Monkhorst-Pack scheme [29] by $(7 \times 7 \times 1)$ mesh. Atomic positions were optimized using the conjugate gradient method, where the total energy and atomic forces were minimized. The energy convergence value between two consecutive steps was chosen as $10^{-5} \mathrm{eV}$ and a maximum force of $0.002 \mathrm{eV} / \AA$ was allowed on each atom. A Gaussian-type Fermi-level smearing method is used with a smearing width of $0.01 \mathrm{eV}$. The energy bands of bare, SL, $\mathrm{Bu}-\mathrm{Sb}$ and aW-Sb structures are calculated by using PBE and corrected by the HSE method [30].

\section{BARE SINGLE-LAYER ANTIMONENE PHASES}

Atomic configuration of $\mathrm{Bu}-\mathrm{Sb}$ and $\mathrm{aW}-\mathrm{Sb}$ phases are shown in Fig. 1. Both ab initio calculations of phonon frequencies and high-temperature molecular dynamics calculations [1] have demonstrated that these two phases remain stable. While the $\mathrm{SL}$ buckled antimonene phase, $\mathrm{Bu}-\mathrm{Sb}$, is a semiconductor with a direct band gap of $1.04 \mathrm{eV}$ calculated by using PBE (1.45 eV calculated by HSE correction [30]), aW-Sb has a relatively small direct band gap of $0.16 \mathrm{eV}$ calculated by using PBE (0.34 eV after HSE correction [30]) [1]. Accordingly these two SL structures have band gaps suitable for various electronic applications. In Fig. 2, the electronic band structures 

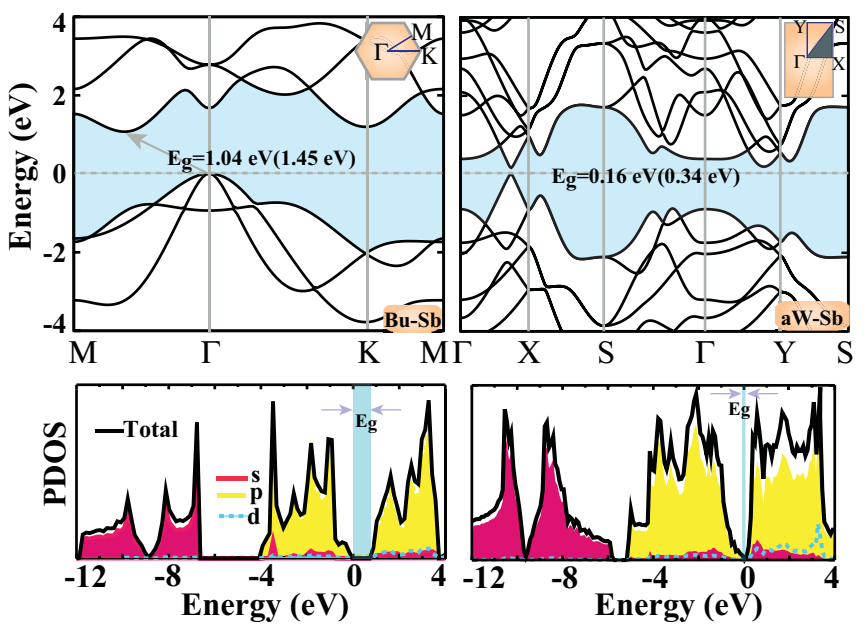

FIG. 2. (a) Electronic energy band structure of the bare SL Bu-Sb antimonene phase and the total and orbital projected densities of states (PDOS). Electronic energies are calculated by PBE. (b) Same for the bare aW-Sb phase. The zero of energy is taken at the top of the valence band. Fundamental band gaps are indicated by arrows. Band gaps calculated using HSE06 correction are shown in parentheses.

of $\mathrm{Bu}-\mathrm{Sb}$ and aW-Sb phases near the fundamental band gap region and the total and orbital projected densities of states (PDOS) in a wider energy range are shown. States originating from $p$ orbitals of $\mathrm{Sb}$ are located at the edges of the valance and conduction bands. Here we note a very special feature of the band structure of the bare aW-Sb phase. The bottom of the conduction and the top of the valence bands occurring at a $\mathbf{k}$ point between the $\mathrm{X}$ and $\Gamma$ points have very high curvature and hence very small effective mass $m^{*}$ implying high carrier mobility. This situation allows easy control of the Fermi level and hence the selection of dominant carrier type in the semiconductor. Additionally, the armchair and zigzag nanoribbons of these single-layer structures have band gaps, which vary with the width of the ribbon. It was also found that the bilayer, as well as the overlayer of antimonene grown on a substrate, is metallized due to the interlayer coupling [1]. While this situation appears to limit the applicability of $\mathrm{Bu}-\mathrm{Sb}$ and $\mathrm{aW}-\mathrm{Sb}$, the undesired interlayer coupling is weakened or lifted by the intercalation molecules as pillars. It is noted that in the previous study [1] the spin-orbit coupling was treated, but nontrivial topological insulator aspects of $R-3 \mathrm{mh}$ quasilayered crystal [31,32], of 2D antimonene phases, and their ribbons were not considered.

\section{ADSORPTION OF ADATOMS TO ANTIMONENE PHASES}

In the adsorption of an adatom, the equilibrium adsorption site, optimized atomic structure and energetics, resulting local electronic and magnetic states, and charge transfer between adatom and substrate have been calculated. Our results are listed in Table I for the adatoms adsorbed to the $\mathrm{Bu}-\mathrm{Sb}$ substrate and in Table II for the adatoms adsorbed to the aW-Sb substrate. In the following sections we examine the details of the adsorption for each adatoms.
TABLE I. Calculated values for an adatom (A) adsorbed to each $(5 \times 5)$ supercell of the SL Bu-Sb phase: the binding energy $E_{b}$, the height of the adatom from the substrate $h$, the smallest distance between the adatom and $\mathrm{Sb}$ atom of antimonene $d_{\mathrm{A}-\mathrm{Sb}}$, the local magnetic moment $\mu$, the charge transfer between the adatom and substrate $\Delta \rho$.

\begin{tabular}{lcrccr}
\hline \hline Adatom $(\mathrm{A})$ & $E_{b}(\mathrm{eV})$ & $h(\AA)$ & $d_{\mathrm{A}-\mathrm{Sb}}(\AA)$ & $\mu\left(\mu_{B}\right)$ & $\Delta \rho(e)$ \\
\hline $\mathrm{H}$ & 1.25 & 1.83 & 1.78 & 1.0 & 0.297 \\
$\mathrm{Li}$ & 1.41 & 1.27 & 2.79 & 0.0 & -0.218 \\
$\mathrm{~B}$ & 4.04 & -1.00 & 2.19 & 1.0 & 1.235 \\
$\mathrm{C}$ & 7.71 & -0.85 & 2.16 & 2.0 & 1.535 \\
$\mathrm{~N}$ & 1.87 & 0.81 & 2.01 & 0.0 & 1.309 \\
$\mathrm{O}$ & 3.46 & 0.54 & 2.00 & 0.0 & 0.957 \\
$\mathrm{Al}$ & 1.66 & 1.74 & 2.87 & 1.0 & -0.281 \\
$\mathrm{Si}$ & 1.93 & 1.30 & 2.77 & 2.0 & 0.235 \\
$\mathrm{P}$ & 1.30 & 1.48 & 2.40 & 1.0 & 0.240 \\
$\mathrm{Cl}$ & 2.26 & 1.99 & 2.98 & 0.0 & 0.630 \\
$\mathrm{Ti}$ & 3.38 & -0.71 & 2.66 & 2.0 & -0.316 \\
$\mathrm{As}$ & 1.22 & 1.48 & 2.51 & 1.0 & 0.481 \\
$\mathrm{In}$ & 1.59 & 2.33 & 3.06 & 1.0 & -0.342 \\
$\mathrm{Sb}$ & 1.19 & 1.66 & 2.76 & 1.0 & 0.0 \\
\hline \hline
\end{tabular}

\section{A. Optimized structure and energetics of adatoms}

All atoms treated in this paper engage in rather strong chemical interaction with $\mathrm{SL} \mathrm{Bu}-\mathrm{Sb}$ and $\mathrm{aW}-\mathrm{Sb}$ phases and form strong bonds. Upon the chemisorption of these adatoms, local deformations and even reconstructions in the crystal structure of the substrate can occur. Here we first consider the adsorption of adatoms to the $\mathrm{Bu}-\mathrm{Sb}$ phase. The optimized atomic structures of adatoms adsorbed to the Bu-Sb substrate are shown in Fig. 3, where local modifications in the atomic configurations around the adatom are described. The binding energies of adatoms such as $\mathrm{H}, \mathrm{Li}, \mathrm{Al}, \mathrm{P}, \mathrm{In}, \mathrm{As}$, and $\mathrm{Sb}$ are in the range of $1-1.7 \mathrm{eV}$. Accordingly, the bonds are not so strong and are formed near the T site $(\mathrm{H}, \mathrm{P}, \mathrm{As}$, and $\mathrm{Sb}$

TABLE II. Calculated values for an adatom (A) adsorbed to each $(4 \times 4)$ supercell of the SL aW-Sb phase: the binding energy $E_{b}$, the height of the adatom from the substrate $h$, the smallest distance between the adatom and $\mathrm{Sb}$ atom of antimonene $d_{\mathrm{A}-\mathrm{Sb}}$, the local magnetic moment $\mu$, the charge transfer between the adatom and substrate $\Delta \rho$.

\begin{tabular}{lrrrrr}
\hline \hline Adatom $(\mathrm{A})$ & $E_{b}(\mathrm{eV})$ & $h(\AA)$ & $d_{\mathrm{A}-\mathrm{Sb}}(\AA)$ & $\mu\left(\mu_{B}\right)$ & $\Delta \rho(e)$ \\
\hline $\mathrm{H}$ & 1.24 & 0.16 & 1.90 & 0.0 & 0.726 \\
$\mathrm{Li}$ & 1.61 & 1.36 & 2.79 & 1.0 & -0.630 \\
$\mathrm{~B}$ & 4.63 & -0.84 & 2.28 & 0.0 & 0.952 \\
$\mathrm{C}$ & 3.95 & 0.40 & 2.07 & 0.0 & 1.185 \\
$\mathrm{~N}$ & 3.41 & 0.26 & 2.02 & 0.0 & 1.245 \\
$\mathrm{O}$ & 4.38 & 0.74 & 2.00 & 0.0 & 1.368 \\
$\mathrm{Al}$ & 2.13 & 1.35 & 2.78 & 0.0 & -0.451 \\
$\mathrm{Si}$ & 2.85 & 1.20 & 2.57 & 0.0 & 0.277 \\
$\mathrm{P}$ & 2.45 & 1.45 & 2.44 & 0.0 & 0.304 \\
$\mathrm{Cl}$ & 2.75 & 2.59 & 2.59 & 0.0 & 0.952 \\
$\mathrm{Ti}$ & 3.06 & 1.15 & 2.68 & 2.0 & -0.893 \\
$\mathrm{As}$ & 2.13 & 0.92 & 2.55 & 0.0 & 0.270 \\
$\mathrm{In}$ & 1.91 & 2.09 & 3.04 & 0.0 & -0.359 \\
$\mathrm{Sb}$ & 1.62 & 1.26 & 2.73 & 0.0 & -0.382 \\
\hline \hline
\end{tabular}



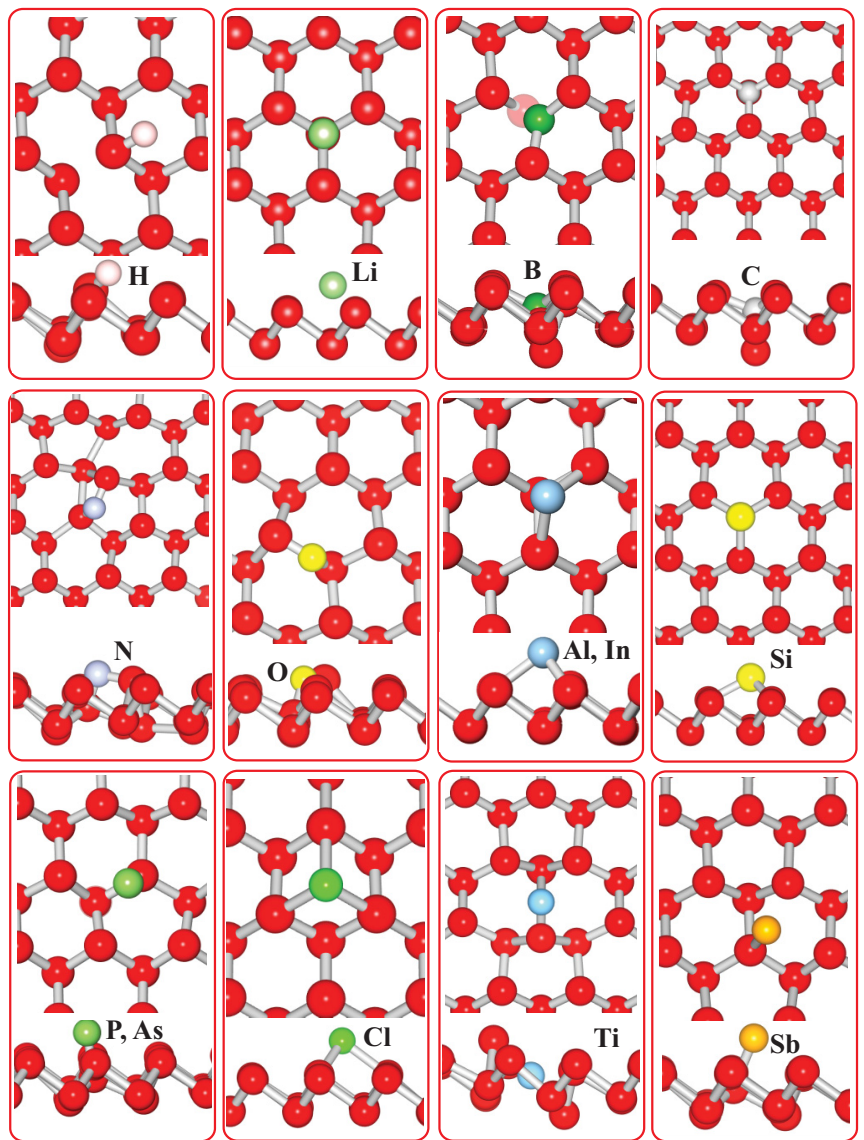

FIG. 3. Top and side views of equilibrium (optimized) atomic structure of the SL Bu-Sb phase after the adsorption of adatoms. While $\mathrm{Sb}$ atoms of the $\mathrm{Bu}-\mathrm{Sb}$ structure are shown by red balls, adsorbed adatoms are presented by balls with different colors.

adatoms) or above the $\mathrm{V}$ site ( $\mathrm{Li}, \mathrm{Al}$, and In adatoms). On the other hand, the binding energies range from $1.9 \mathrm{eV}$ to $7.7 \mathrm{eV}$ and local reconstructions can take place when $\mathrm{B}, \mathrm{C}, \mathrm{N}$, $\mathrm{O}, \mathrm{Si}$, and $\mathrm{Ti}$ adatoms are adsorbed to $\mathrm{Bu}-\mathrm{Sb}$. According to the Bader analysis [33], $\mathrm{Li}, \mathrm{Al}, \mathrm{Ti}$, and In donate charge to the $\mathrm{Sb}$ substrate, and the rest of the adatoms receive charge from the Sb substrate.

Some of the critical adsorption geometries and resulting local reconstructions are summarized: The boron atom substitutes the $\mathrm{Sb}$ atom at the $\mathrm{V}$ site by pushing it downwards and acquires 1.24 electrons from the substrate. Like the B atom, the $\mathrm{C}$ atom also substitutes the $\mathrm{Sb}$ atom at the $\mathrm{V}$ site by pushing it downwards. The binding energies of $\mathrm{B}$ and $\mathrm{C}$ adatoms are rather high and $4.04 \mathrm{eV}$ and $7.71 \mathrm{eV}$, respectively. The nitrogen atom, in the same row as B and C, behaves differently. It is attached to a Br-site by inducing a Stone-Wales type defect in the substrate [34]. As for the $\mathrm{O}$ adatom, it is attached to the substrate in a position between the $\mathrm{T}$ and $\mathrm{Br}$ sites and deforms the hexagon rings. Silicon is adsorbed at the $\mathrm{T}$ site by pushing the $\mathrm{Sb}$ atom underneath. This configuration is reminiscent of the dumbbell structure in silicene and germanene [16]. The chlorine adatom is adsorbed above the $\mathrm{V}$ site. The titanium adatom induces also a local reconstruction, where it stretches the $\mathrm{Sb}-\mathrm{Sb}$ bond and forms an Sb-Ti-Sb bond; it donates a 0.316 electron charge to the substrate and attains a binding energy of $3.38 \mathrm{eV}$.
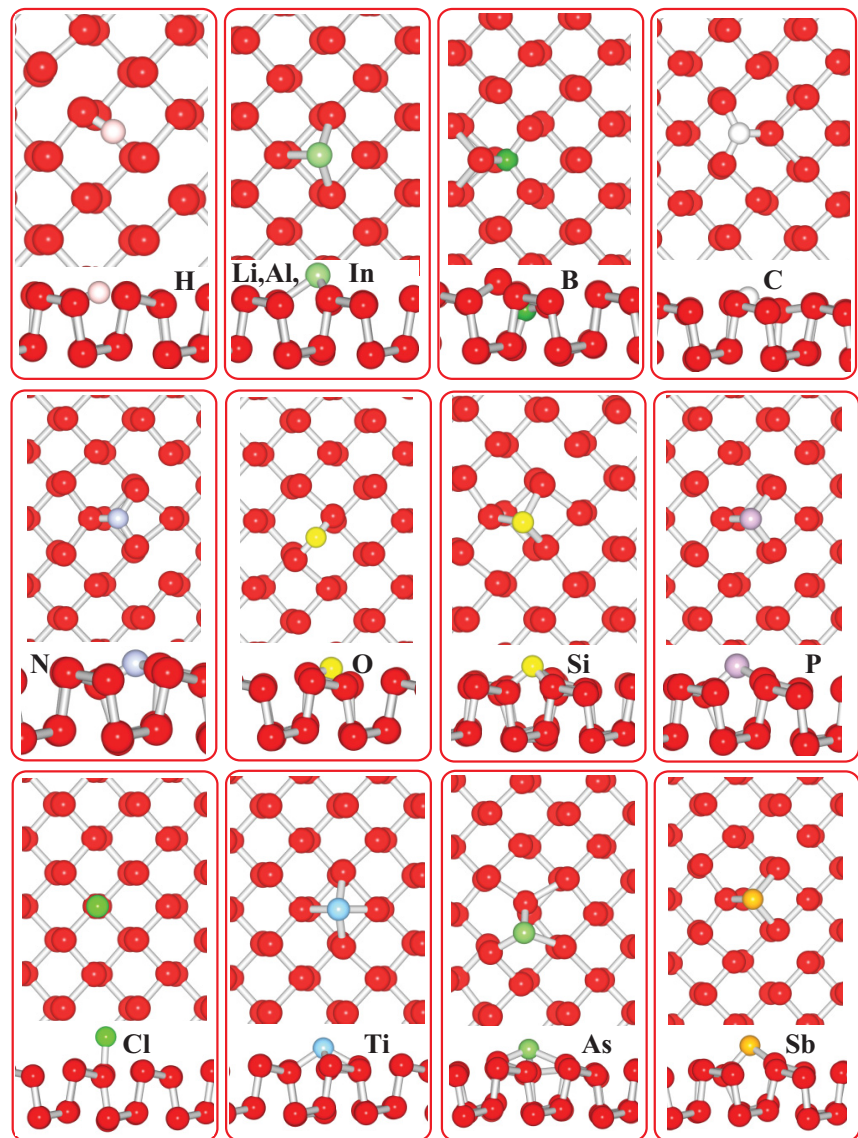

FIG. 4. Top and side views of equilibrium (optimized) atomic structure of the SL aW-Sb phase after the adsorption of adatoms. While $\mathrm{Sb}$ atoms of the aW-Sb structure are shown by red balls, adsorbed adatoms are presented by balls with different colors.

Optimized atomic configurations of adatoms adsorbed to the aW-Sb phase are shown in Fig. 4. All adatoms except $\mathrm{B}$ stayed above the upper layer of aW-Sb. As an exception, the $\mathrm{B}$ adatom by itself is implemented into the aW-Sb by pushing one $\mathrm{Sb}$ atom on the upper plane and forms strong bonds with substrate atoms. Due to changes in the adsorption geometry also the charge transfer between adatom and substrate undergoes changes. The atomic structure of $\mathrm{aW}-\mathrm{Sb}$ appears to be more robust under adsorption of adatoms; severe reconstructions do not take place. For example, while the $\mathrm{C}$ adatom gives rise to the reconstruction on the $\mathrm{Bu}-\mathrm{Sb}$ substrate, the $\mathrm{C}$ adatom stayed slightly above aW-Sb and any severe reconstruction is hindered by an energy barrier. Hence aW-Sb remained rather stable upon the adsorption of $\mathrm{C}$ and accordingly, the binding energy of the $\mathrm{C}$ adatom to $\mathrm{aW}-\mathrm{Sb}$ becomes $\sim 3 \mathrm{eV}$ smaller than that on $\mathrm{Bu}-\mathrm{Sb}$. However, the binding energies of $\mathrm{N}, \mathrm{O}, \mathrm{Al}, \mathrm{Si}, \mathrm{P}$, and $\mathrm{As}$ to $\mathrm{aW}-\mathrm{Sb}$ increased relative to that of $\mathrm{Bu}-\mathrm{Sb}$ due to more favorable adsorption geometries. As we discuss in forthcoming sections, due to differences in the adsorption geometry the electronic and magnetic structure of aW-Sb differ from $\mathrm{Bu}-\mathrm{Sb}$.

\section{B. Electronic and magnetic properties}

Adatoms can modify the electronic and magnetic structure of the $\mathrm{Bu}-\mathrm{Sb}$ and $\mathrm{aW}-\mathrm{Sb}$ phases. In the doping at very low 
coverage, single adsorbed or substituted foreign atoms give rise to localized states in the band gap or resonance states in the band continua of the Bu-Sb and aW-Sb phases. In this study we are concerned with the local effects on the electronic structure of the $\mathrm{Bu}-\mathrm{Sb}$ and aW-Sb substrates attained by the doping of adatoms. Since the adsorption is treated by relatively large $(n \times n)$ supercells, the adatom-adatom coupling is generally minute and hence our model can mimic the isolated dopant.

Here we followed a scheme to deduce the effects of adsorption, where the energy positions of dopant or adatom induced localized states relative to the rest of the substrate and energy shifts can be revealed. Accordingly, rather than the energy bands of the supercells, here we investigate the total and adatom projected densities of states to reveal the energy locations of dopant states in the fundamental band gap of the extended substrate as shown in Figs. 5 and 6. The density of states of the extended substrate and the energy position of its fundamental band gap is determined by the local density of states (i.e., the projected total density of states) at a substrate $\mathrm{Sb}$ atom, which is farthest to the adatom. This way, the energy positions of adatom-induced localized states can be determined relative to the band edges of the extended substrate. The common Fermi level is normally within the fundamental band gap; it can move towards the band edges depending on the character of the localized states. This way energy shifts of localized adatom states, which are induced due to charge exchange between adatom and substrate, can be deduced with reasonable accuracy. In Figs. 5 and 6, the densities of states of the extended substrate are shown by gray distribution, while the fundamental band gap $E_{g}$ between the band edges of the extended substrate is highlighted by a light blue zone. In what follows, we examine the features of calculated electronic and magnetic properties.

The features of a single adatom adsorbed to $\mathrm{Bu}-\mathrm{Sb}$ are presented in Fig. 5. States derived from a hydrogen adatom adsorbed to $\mathrm{Bu}-\mathrm{Sb}$ are spin polarized and appear near the edge of the valence band. The Li adatom donates part of its valence charge to the excited state, which overlaps with the bottom of the conduction band of the extended substrate. This situation implies that the adatom+substrate system is metallized at high coverage of Li. Spin-polarized unoccupied localized states induced by B are located in the midgap as well as near the conduction band edge of the extended substrate. The carbon adatom displays a similar situation; localized states induced by the $\mathrm{C}$ adatom are $\sim 0.3 \mathrm{eV}$ below the edge of the conduction band edge. While $\mathrm{N}$ induces unoccupied, localized states occurring at the band edges, hence decreasing the band gap, the resonance states derived from the $\mathrm{O}$ adatom are located in band continua. Aluminum gives rise to donor states just below the conduction band edge; the occupied spin-up states of $\mathrm{Si}$ at the midgap have a small band gap from its spin-down states. The occupied, localized spin-up states of P occur near the edge of the valence band. In the case of $\mathrm{Cl}$, since the Fermi level shifts down from the valence band edge due to the finite size of the supercell, eventually the hole conductivity is favored. Spin-up and spin-down states of Ti with small energy splitting occur in the band gap. The energy locations of spin-polarized states and their integer magnetic moments $\left(\mu=2 \mu_{B}\right)$ of $\mathbf{C}, \mathbf{S i}$,

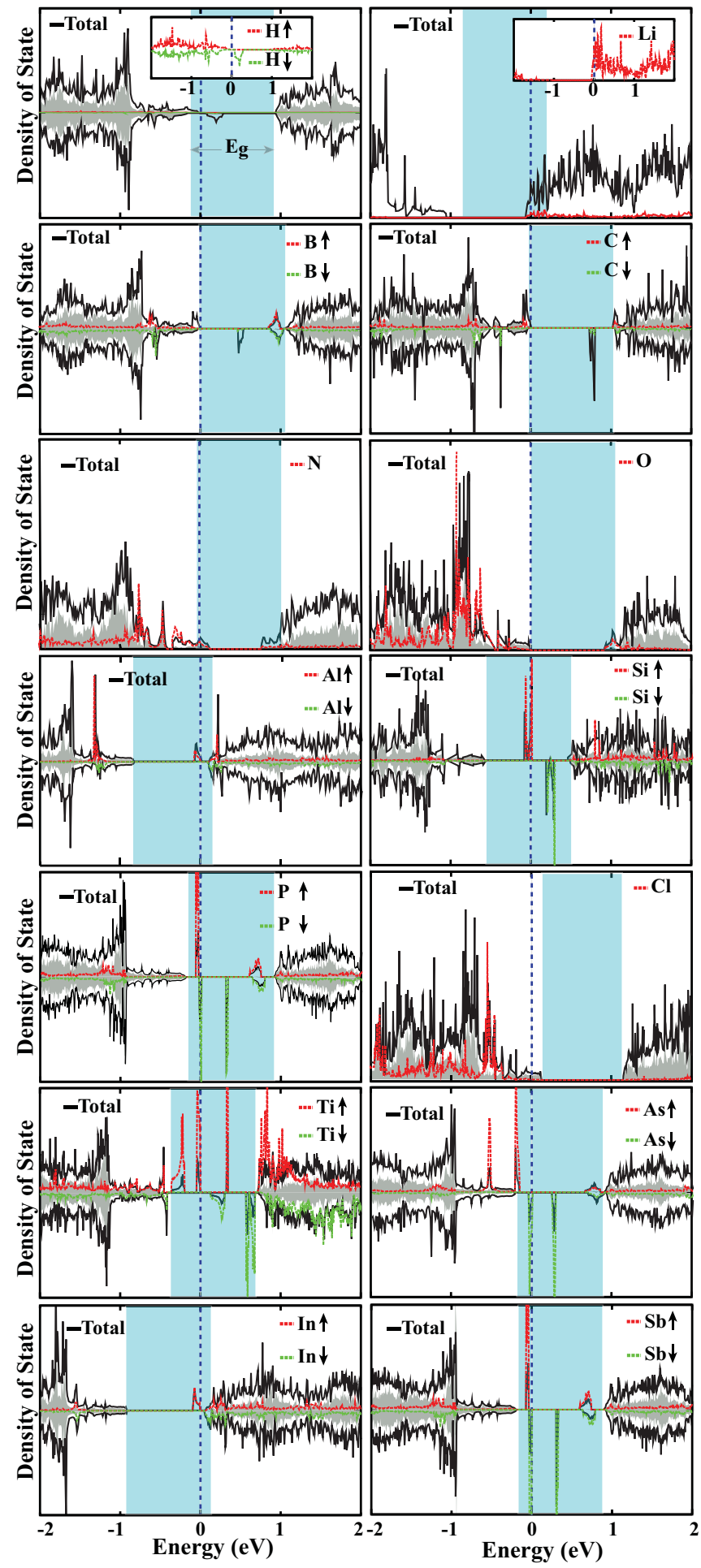

FIG. 5. Total (shown by black lines) and adatom projected (shown by red and green lines) densities of states for a single adatom adsorbed to each $(5 \times 5)$ supercell of the $\mathrm{Bu}-\mathrm{Sb}$ phase. The density of states of the extended $\mathrm{Bu}-\mathrm{Sb}$ substrate shown in gray tone is obtained from the local density of states calculated at a host $\mathrm{Sb}$ atom farthest from the adatom; its band gap is shown by the light blue zones. The zero of the energy is set at the common Fermi level shown by dashed vertical line. For clarity, the projected densities of states of specific adatoms are shown by insets. 

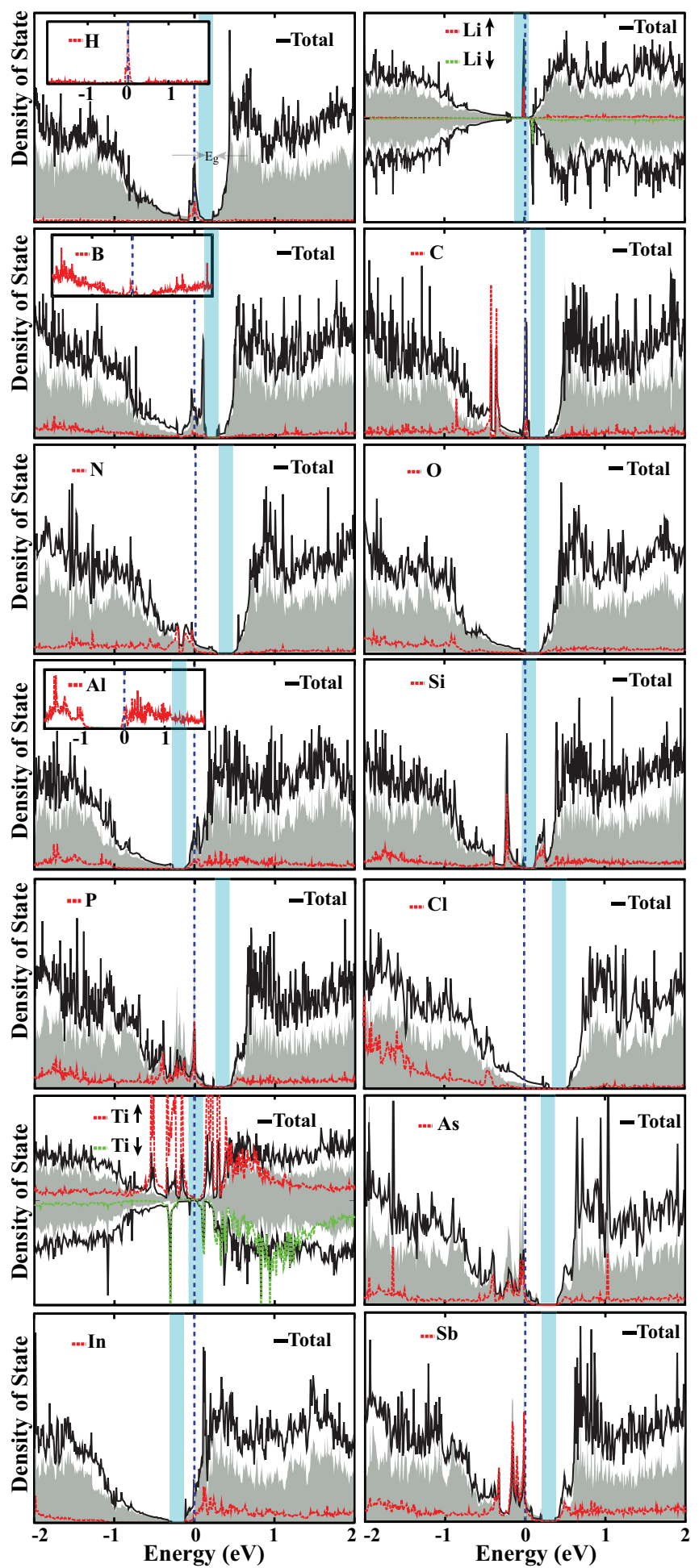

FIG. 6. Total (shown by black lines) and adatom projected (shown by red and green lines) densities of states for a single-adatom adsorbed to each $(4 \times 4)$ supercell of the aW-Sb substrate. The density of states of extended $\mathrm{Bu}-\mathrm{Sb}$ substrate shown in gray tone is obtained from the local density of states calculated at a host $\mathrm{Sb}$ atom farthest from the adatom; its band gap is shown by the light blue color. The zero of the energy is set at the common Fermi level shown by the dashed vertical line. For clarity, the projected densities of states of specific adatoms are shown by insets. and $\mathrm{Ti}$ adatoms suggest that $\mathrm{Bu}-\mathrm{Sb}$ can attain half metallicity at high coverage of one of these adatoms. Arsenic and $\mathrm{Sb}$ adatoms behave similarly: their occupied spin-up states near the valence band edge and unoccupied spin-down states at the midgap. Indium induces only a donor state near the edge of the conduction band. Briefly, the adatoms treated in the present study add diversity of electronic features to the electronic properties of $\mathrm{Bu}-\mathrm{Sb}$.

The effects of single adatoms adsorbed to the aW-Sb substrate are illustrated in Fig. 6. The extended substrate has a small band gap. Additionally, as pointed out in Sec. III, the top of the valence and the bottom of the conduction bands occur at the same $\mathbf{k}$ point between the $X$ and $\Gamma$ points and have very high curvatures. Hence, they have low state densities and small effective masses $m^{*}$. Consequently, the Fermi level can easily go outside of the fundamental band gap in finite systems treated within the supercell method, if the states originating from adatoms occur at the edges of valance and conduction band and are filled by the electrons of the substrate or vise versa. The situation in Fig. 6 complies with the calculated charge transfer values $\Delta \rho$ in Table II. This is an artifact of the finite-size supercell model, which becomes critical for the bare aW-Sb phase having a special band structure. We will discuss the electronic and magnetic properties of adatoms adsorbed to aW-Sb by taking this situation into account.

Adsorbed $\mathrm{H}$ has occupied states near the edge of the valence band and unoccupied states below the edge of the conduction band of the extended aW-Sb substrate. Occupied spin-up and empty spin-down states originating from $\mathrm{Li}$ occur near the edge of the conduction band. Having an integer magnetic moment, aW-Sb covered by Li may display half-metallic features. Similarly to the Bu-Sb substrate, B and $\mathrm{C}$ lead to states near the edge of the valence band. $\mathrm{Si}, \mathrm{P}, \mathrm{Cl}, \mathrm{As}$, and $\mathrm{Sb}$ adatoms are nonmagnetic and have states near the valence band edge. In contrast, In and $\mathrm{Al}$ donate charge to the $\mathrm{aW}-\mathrm{Sb}$ and give rise to states overlapping with the conduction band. Hence they behave as if donor atoms. Titanium is spin polarized and gives rise to several spin-up and spin-down states originating its $3 d$ orbitals. These states occur at both band edges. The calculated magnetic moment Ti adatom is integer and $\mu=2.0$ $\mu_{B}$. Like Li, this situation may attribute half-metallic character to aW-Sb covered by Ti.

It should be noted that for the energies of excited states of $\mathrm{Bu}-\mathrm{Sb}$, in particular, the band gap is slightly underestimated by calculations using PBE. For example, the PBE band gap is raised from $104 \mathrm{eV}$ to $1.45 \mathrm{eV}$ upon the HSE correction [30]. Under these circumstances, the energy positions of unoccupied adatom derived states relative to the actual conduction band edge can be affected slightly. This effect can be even smaller if the same scissor operation of bare $\mathrm{Sb}$ substrates is also applicable to the adatom+Sb substrate system. However, the energies of those states below the Fermi level corresponding to the ground state of the adatom-substrate system and resulting charge exchange can be predicted by PBE calculations with reasonable accuracy. The shortcoming of PBE calculations may be even less serious for the gap states derived from adatoms adsorbed to $\mathrm{aW}-\mathrm{Sb}$, since the fundamental band gap is narrow and underestimated by only $0.18 \mathrm{eV}$ by PBE in the present study. 


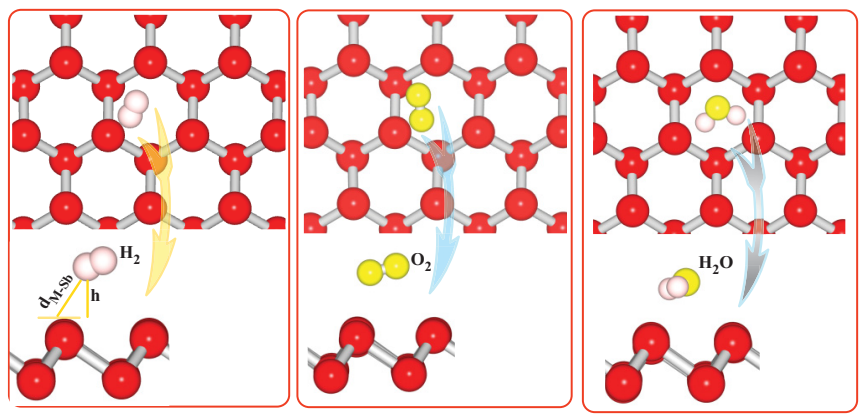

FIG. 7. Top and side view of the schematic representation of $\mathrm{H}_{2}$, $\mathrm{O}_{2}$, and $\mathrm{H}_{2} \mathrm{O}$ molecules adsorbed to each $5 \times 5$ supercell of $\mathrm{Bu}-\mathrm{Sb}$.

\section{PHYSISORPTION OF MOLECULES TO Bu-Sb STRUCTURE}

The physisorption of three molecules, $\mathrm{H}_{2}, \mathrm{O}_{2}$, and $\mathrm{H}_{2} \mathrm{O}$, to the $\mathrm{Bu}-\mathrm{Sb}$ phase has been investigated. First, each molecule is placed in diverse configurations above various possible physisorption sites at a large distance from the substrate. Then the atomic positions of the whole system, namely molecule and substrate atoms, are optimized to attain minimum total energy, as well as atomic forces. The optimized structures corresponding to minimum total energy are presented in Fig. 7. Calculated values regarding the binding energies and molecule-substrate distances are listed in Table III.

The optimized atomic configuration and calculated values related with energetics and physisorption configuration indicate that the molecule-substrate interaction is rather weak. For example, the binding energy of $\mathrm{H}_{2}$ is $124 \mathrm{meV}$, which is rather low and is typical for the van der Waals interaction. The situations for $\mathrm{O}_{2}$ and $\mathrm{H}_{2} \mathrm{O}$ are also similar with binding energies $119 \mathrm{meV}$ and $227 \mathrm{meV}$, respectively. As for the heights of molecules and the minimum distances between molecule and substrate $\mathrm{Sb}$ atom, they are rather large in compliance with low binding energies. Accordingly, the bare $\mathrm{Bu}-\mathrm{Sb}$ phase is rather inert to the molecules $\mathrm{H}_{2}, \mathrm{O}_{2}$, and $\mathrm{H}_{2} \mathrm{O}$. In this respect, dissociation of these molecules cannot take place on $\mathrm{Bu}-\mathrm{Sb}$ in normal conditions. Edges, vacancy defects, or adsorbed adatoms of $\mathrm{Bu}-\mathrm{Sb}$ may be possible places which mediate dissociation of these molecules. Notably, specific adatoms are expected to form relatively stronger bonds with some of these molecules. For example, as outlined in Sec. IV, Ti forms strong bonds with $\mathrm{Bu}-\mathrm{Sb}$. It is known that the adsorbed Ti being a $3 d$-transition-metal element can bind four $\mathrm{H}_{2}$ molecules with a special Kubas [35] bonding for high-capacity hydrogen storage on single-walled carbon nanotubes and

TABLE III. Calculated values for the optimized structure of the single molecule $(\mathrm{M})$ adsorbed to $(5 \times 5)$ supercell of the $\mathrm{Bu}-\mathrm{Sb}$ phase. $E_{a}$, the physisorption energy of the molecule; $h$, the height of the molecule from the original atomic plane of substrate; $d_{\mathrm{M}-\mathrm{Sb}}$, the minimum distance between the molecule and the substrate $\mathrm{Sb}$ atom.

\begin{tabular}{lccc}
\hline \hline Molecule $(\mathrm{M})$ & $E_{a}(\mathrm{eV})$ & $h(\AA)$ & $d_{\mathrm{M}-\mathrm{Sb}}(\AA)$ \\
\hline $\mathrm{H}_{2}$ & 0.124 & 3.09 & 3.40 \\
$\mathrm{O}_{2}$ & 0.119 & 2.61 & 3.76 \\
$\mathrm{H}_{2} \mathrm{O}$ & 0.227 & 2.44 & 2.93 \\
\hline \hline
\end{tabular}

TABLE IV. Calculated values for the optimized structure of the single molecule $(\mathrm{M})$ adsorbed to $(4 \times 4)$ supercell of the $\mathrm{aW}-\mathrm{Sb}$ phase. $E_{a}$, the physisorption energy of the molecule; $h$, the height of the molecule from the original atomic plane of substrate; $d_{\mathrm{M}-\mathrm{Sb}}$, the minimum distance between the molecule and the substrate $\mathrm{Sb}$ atom.

\begin{tabular}{lccc}
\hline \hline Molecule $(\mathrm{M})$ & $E_{a}(\mathrm{eV})$ & $h(\AA)$ & $d_{\mathrm{M}-\mathrm{Sb}}(\AA)$ \\
\hline $\mathrm{H}_{2}$ & 0.124 & 2.80 & 3.30 \\
$\mathrm{O}_{2}$ & 0.254 & 2.61 & 3.27 \\
$\mathrm{H}_{2} \mathrm{O}$ & 0.225 & 2.48 & 2.79 \\
\hline \hline
\end{tabular}

graphene $[10,11]$. A similar situation is expected to occur for the antimonene phases.

In Table IV the values of the binding energy and relevant structural parameters calculated for the molecules $\mathrm{H}_{2}, \mathrm{O}_{2}$, and $\mathrm{H}_{2} \mathrm{O}$ physisorbed to the aW-Sb substrate are presented. Similarly to $\mathrm{Bu}-\mathrm{Sb}$, weak binding energies and large heights are also attained on $\mathrm{aW}-\mathrm{Sb}$, indicating van der Waals interaction between the molecules and the aW-Sb substrate.

\section{CONCLUSIONS}

In this paper we investigated the interaction between single adatoms and molecules with the buckled $\mathrm{Bu}-\mathrm{Sb}$ and asymmetric washboard aW-Sb phases of antimonene. While our calculations and analysis are focused to the single, isolated adatoms adsorbed and molecules physisorbed to antimonene, our results provide valuable information pertaining to the functionalization of antimonene in various applications. Even if the interactions of molecules such as $\mathrm{H}_{2}, \mathrm{O}_{2}$, and $\mathrm{H}_{2} \mathrm{O}$ with nanomaterials are crucial for hydrogen storage, hydrogen evaluation reaction, and oxidation-deoxidation processes, these molecules weakly interact with antimonene; their binding energies are very small and have predominantly van der Waals character. However, selected adatoms can form rather strong bonds with antimonene by exchanging electronic charges and cause local reconstructions and defects. The electronic states originating from these adatoms give rise to diversity of electronic states. In particular, aW-Sb having high-curvature bands at the edges of fundamental band gaps can attain high carrier mobility, once it is doped with specific atoms. Some of the adatoms acquire magnetic moments through their spin-polarized electronic states. While bare antimonene phases are nonmagnetic semiconductors, they can acquire magnetism through decoration with selected adatoms deduced in this paper. That the magnetic moments are integer multiples of the Bohr magneton suggests that antimonene can be a half metal upon decoration with some adatoms.

\section{ACKNOWLEDGMENTS}

The computational resources are provided by TUBITAK ULAKBIM, High Performance and Grid Computing Center (TR-Grid e-Infrastructure). O.Ü.A., E.A., and S.C. acknowledge financial support from the Academy of Sciences of Turkey (TÜBA). This study was performed while O.Ü.A. and E.A. were visiting the Department of Physics, Bilkent University. 
[1] O. Ü. Aktürk, V. O. Özçelik, and S. Ciraci, Phys. Rev. B 91, 235446 (2015).

[2] E. Durgun, S. Tongay, and S. Ciraci, Phys. Rev. B 72, 075420 (2005).

[3] S. Cahangirov, M. Topsakal, E. Aktürk, H. Sahin, and S. Ciraci, Phys. Rev. Lett. 102, 236804 (2009).

[4] S. Cahangirov, M. Topsakal, and S. Ciraci, Phys. Rev. B 81, 195120 (2010).

[5] H. Sahin, S. Cahangirov, M. Topsakal, E. Bekaroglu, E. Aktürk, R. T. Senger, and S. Ciraci, Phys. Rev. B 80, 155453 (2009).

[6] Z. Zhu and D. Tomanek, Phys. Rev. Lett. 112, 176802 (2014); M. Liu, A. T. Neal, Z. Zhu, Z. Luo, X. Xu, D. Tomanek, and P. D. Yu, ACS Nano 8, 4033 (2014).

[7] C. Maanit, A. McKinley, and R. H. Williams, J. Phys. C 18, 4975 (1985).

[8] M. W. Grant, P. F. Lyman, J. H. Hoogenraad, and L. E. Seiberling, Surf. Sci. 279, 180 (1992).

[9] R. Whittle, I. T. McGovern, D. R. T. Zahn, C. Müller, C. Nowak, A. Cafolla, and W. Braun, Appl. Surf. Sci. 56, 218 (1992).

[10] T. Yildirim and S. Ciraci, Phys. Rev. Lett. 94, 175501 (2005).

[11] E. Durgun, S. Ciraci, W. Zhou, and T. Yildirim, Phys. Rev. Lett. 97, 226102 (2006).

[12] C. Ataca, E. Aktürk, S. Ciraci, and H. Ustunel, Appl. Phys. Lett. 93, 043123 (2008).

[13] H. Sevincli, M. Topsakal, E. Durgun, and S. Ciraci, Phys. Rev. B 77, 195434 (2008).

[14] C. Ataca, E. Aktürk, H. Sahin, and S. Ciraci, J. Appl. Phys. 109, 013704 (2011).

[15] C. Ataca, E. Aktürk, and S. Ciraci, Phys. Rev. B 79, 041406 (2009).
[16] V. O. Özçelik, H. H. Gurel, and S. Ciraci, Phys. Rev. B 88, 045440 (2013).

[17] T. P. Kaloni and U. Schwingenschlögl, Phys. Status Solidi RRL 8, 685 (2014).

[18] Y. Ding, Y. Wang, L. Shi, Z. Xu, and J. Ni, Phys. Status Solidi RRL 8, 939 (2014).

[19] C. Ataca and S. Ciraci, J. Phys. Chem. C 115, 27 (2011).

[20] M. Topsakal and S. Ciraci, Phys. Rev. B 86, 205402 (2012).

[21] M. Topsakal, H. H. Gurel, and S. Ciraci, J. Phys. Chem. C 117, 5943 (2013).

[22] H. H. Gurel and S. Ciraci, J. Phys.: Condens. Matter 25, 435304 (2013).

[23] C. Ataca and S. Ciraci, Phys. Rev. B 85, 195410 (2012).

[24] W. Kohn and L. J. Sham, Phys. Rev. 140, A1133 (1965).

[25] S. Grimme, J. Comput. Chem. 27, 1787 (2006).

[26] P. E. Blöchl, Phys. Rev. B 50, 17953 (1994).

[27] J. P. Perdew, K. Burke, and M. Ernzerhof, Phys. Rev. Lett. 77, 3865 (1996).

[28] G. Kresse and J. Furthmüller, Phys. Rev. B 54, 11169 (1996).

[29] H. J. Monkhorst and J. D. Pack, Phys. Rev. B 13, 5188 (1976).

[30] J. Heyd, G. E. Scuseria, and M. Ernzerhof, J. Chem. Phys. 118, 8207 (2003).

[31] J. E. Moore, Nature (London) 464, 194 (2010).

[32] I. Aguilera, C. Friedrich, and S. Blügel, Phys. Rev. B 91, 125129 (2015).

[33] G. Henkelman, A. Arnaldsson, and H. Jonsson, Comput. Mater. Sci. 36, 354 (2006).

[34] A. J. Stone and D. J. Wales, Chem. Phys. Lett. 128, 501 (1986).

[35] Metal Dihydrogen and Bond Complexes: Structure, Theory, and Reactivity, edited by G. J. Kubas (Kluwer Academics/Plenum Publishing, New York, 2001). 